\title{
A influência do Zinco, Selênio e Iodo na suplementação alimentar em pessoas com Hipotireoidismo
}

The influence of Zinc, Selenium and Iodine on dietary supplementation in people with Hypothyroidism

La influencia del Zinc, el Selenio y el Yodo en la suplementación dietética en personas con Hipotiroidismo

Liana Cynthia de Macedo Reis

ORCID: https://orcid.org/0000-0001-7304-7713 Instituto Federal de Educação, Ciências e Tecnologia do Piauí, Brasil E-mail: lianareis@ifpi.edu.br Francisco Laurindo da Silva ORCID: https://orcid.org/0000-0001-6837-4509 Centro Universitário UniFacid, Brasil E-mail: flspb@yahoo.com.br Amanda Laurindo Monteiro

ORCID: https://orcid.org/0000-0002-4832-4544 Centro Universitário UniFacid, Brasil

E-mail: amandalaurindo_@hotmail.com

Marcos André Arrais de Sousa

ORCID: https://orcid.org/0000-0002-7652-0198 Centro Universitário UniFacid, Brasil E-mail: marcosarraiss007@gmail.com

Ana Graziela Soares Rêgo Lobão ORCID: https://orcid.org/0000-0003-3003-954X Centro Universitário UniFacid, Brasil E-mail: grazielarego@ hotmail.com

Rilkaelle Gomes de Melo Cerqueira ORCID: https://orcid.org/0000-0002-0835-9515 Centro Universitário UniFacid, Brasil E-mail: rilkaelle.cerqueira@gmail.com

Suzana Christiany Pontes Carneiro ORCID: https://orcid.org/0000-0002-0881-0441 NovaUnesc, Brasil

E-mail: suzanacpontes@gmail.com

Sergio Augusto Dias Castro

ORCID: https://orcid.org/0000-0003-4064-9341 Instituto Federal de Educação, Ciências e Tecnologia do Paraná, Brasil E-mail: sergio.castro@ifpr.edu.br

\begin{abstract}
Resumo
O presente estudo teve como objetivo identificar a influência do zinco, selênio e iodo no controle do hipotireoidismo subclínico e suas descrições na literatura científica, que são detentoras de um grande potencial de inovação e ainda não foram exploradas em todas as suas potencialidades. Foi realizada uma revisão de literatura, no qual adota um processo replicável, científico e transparente, sendo recomendada para reunir e analisar os estudos relevantes sobre o tema. Cinco bases de dados científicas foram utilizadas na pesquisa, ScienceDirect Journals (Elsevier), Directory of Open Access Journals (DOAJ), PMC (PubMed Central), MEDLINE/PubMed (NLM) e Scielo. Os termos indexadores foram utilizados na língua inglesa "hypothyroidism" e "food". Como critério de inclusão foi utilizado artigos que tinham um dos termos indexadores no título e/ou resumo e/ou palavras-chave, publicados nos últimos 5 anos. Após a busca nas bases de dados, identificou 70 artigos e após o procedimento de filtragem e seleção resultou em 19 artigos selecionados para o presente estudo. Uma dieta adequada e balanceada pode se tornar também um fator importante para ajustar o bom funcionamento da glândula tiróide (GT). Observou -se que os minerais, zinco, selênio e iodo, são importantes para a manutenção do organismo e para a produção de hormônios tireoidianos, com isso as quantidades destes componentes no corpo devem ter sempre um equilíbrio.
\end{abstract}

Palavras-chave: Iodo; Selênio; Zinco; Hipotireoidismo. 


\begin{abstract}
The present study aimed to identify the influence of zinc, selenium and iodine in the control of subclinical hypothyroidism and their descriptions in the scientific literature, which have a great potential for innovation and have not yet been fully explored. A literature review was carried out, in which it adopts a replicable, scientific and transparent process, being recommended to gather and analyze relevant studies on the subject. Five scientific databases were used in the research, ScienceDirect Journals (Elsevier), Directory of Open Access Journals (DOAJ), PMC (PubMed Central), MEDLINE/PubMed (NLM) and Scielo. The indexing terms were used in the English language "hypothyroidism" and "food". As inclusion criteria, articles that had one of the indexing terms in the title and/or abstract and/or keywords, published in the last 5 years, were used. After searching the databases, 70 articles were identified and after the filtering and selection procedure resulted in 19 articles selected for the present study. A proper and balanced diet can also become an important factor in adjusting the proper functioning of the thyroid gland (TG). It was observed that minerals, zinc, selenium and iodine, are important for the maintenance of the organism and for the production of thyroid hormones, with this the amounts of these components in the body must always be balanced.
\end{abstract}

Keywords: Iodine; Selenium; Zinc; Hypothyroidism.

\title{
Resumen
}

El presente estudio tuvo como objetivo identificar la influencia del zinc, el selenio y el yodo en el control del hipotiroidismo subclínico y sus descripciones en la literatura científica, los cuales tienen un gran potencial de innovación y aún no han sido completamente explorados. Se realizó una revisión de la literatura, en la que se adopta un proceso replicable, científico y transparente, recomendándose recopilar y analizar estudios relevantes sobre el tema. Se utilizaron cinco bases de datos científicas en la investigación, ScienceDirect Journals (Elsevier), Directory of Open Access Journals (DOAJ), PMC (PubMed Central), MEDLINE / PubMed (NLM) y Scielo. Los términos de indexación se utilizaron en el idioma inglés "hipotiroidismo" y "comida". Como criterio de inclusión se utilizaron artículos que tuvieran alguno de los términos indexados en el título y / o resumen y / o palabras clave, publicados en los últimos 5 años. Tras la búsqueda en las bases de datos, se identificaron 70 artículos y tras el procedimiento de filtrado y selección se obtuvieron 19 artículos seleccionados para el presente estudio. Una dieta adecuada y equilibrada también puede convertirse en un factor importante para ajustar el correcto funcionamiento de la glándula tiroides (TG). Se observó que los minerales, zinc, selenio y yodo, son importantes para el mantenimiento del organismo y para la producción de hormonas tiroideas, por lo que las cantidades de estos componentes en el organismo siempre deben estar equilibradas.

Palabras clave: Yodo; Selenio; Zinc; Hipotiroidismo.

\section{Introdução}

Os hábitos alimentares interferem diretamente na produção de hormônios no corpo humano, especificamente em relação à glândula tireóide, podem-se destacar insumos que atuam tanto positiva quanto negativamente em relação a seu desenvolvimento. Por se tratar de uma glândula endócrina, a tireóide é de extrema importância para o metabolismo e crescimento. A tireóide secreta dois importantes hormônios, a tiroxina (T4) e a tri-iodotironina (T3), ambas com efeito de controlar o crescimento, o metabolismo e o desenvolvimento corporal, desempenhando funções na produção de proteínas estruturais, enzimas e outros hormônios. Para que ocorra a síntese e a função adequada dos hormônios da tireoide (HTs), são requeridos muitos micronutrientes como iodo, selênio e zinco (Chen 2015, Mezzomo 2016, Mahadevan 2017).

O hipotireoidismo é caracterizado por uma deficiência de produção do hormônio pela glândula tiroide, que pode ser severa ou moderada, a dieta é um dos fatores de risco para o surgimento e o agravamento do hipotireoidismo. Uma deficiência grave de hormônios da tiróide se manifesta como hipotireoidismo declarado. A forma moderada denominada hipotireoidismo subclínico (HS) raramente apresenta sinais e sintomas e é definida pela concentração de TSH bioquimicamente acima do limite superior do intervalo de referência, com os níveis de hormônio tireoidiano que permanecem dentro dos limites de referência. O HS deve ser definido na ausência de doença hipotalâmica ou pituitária e de doença não tireoidiana. O hipotireoidismo se refere a uma diminuição da produção do hormônio da tireóide, o que provoca um aumento nos níveis de TSH. O hipotireoidismo pode também ser causado pela ação reduzida dos hormônios tireoidianos nos órgãos-alvo, como nos casos raros de resistência aos hormônios tireoidianos. A causa mais frequente do hipotireoidismo é a tireoidite autoimune crônica (tireoidite de Hashimoto). No entanto, ele também pode resultar do tratamento do hipertireoidismo com cirurgia, radiação com 131-I ou 
após o uso de drogas antitiroidianas. Várias formas de tireoidite, tais como pós-parto, subaguda, silenciosa ou tireoidite induzida por citoquinas, também podem causar hipotireoidismo permanente ou transitório. Menos frequentemente, pode ser uma consequência de doenças infiltrativas ou infecciosas, radioterapia externa, disgenesias da tireóide, defeitos funcionais na biossíntese e liberação dos hormônios tireoidianos ou defeitos congênitos na biossíntese dos hormônios da tireóide. Tanto a deficiência como o excesso de iodo são causas bem reconhecidas de hipotireoidismo (Brenta 2013, Mezzomo 2016).

A prevalência do hipotireoidismo é relatada como de 1,5\%, porém o HS é quase dez vezes mais frequente. A sua prevalência é diferente de acordo com o estudo (entre 6-20\%) dependendo da idade, sexo, raça, iodossuficiencia, da população estudada, bem como do nível de corte do TSH empregado para definir HS. Porém apresenta maior prevalência no sexo feminino, caucasianos e idosos. Estudos em certos países, como a Indonésia, apontam altas dosagens de iodo no sal utilizado pela maioria das pessoas. Além disso, alguns alimentos são, por si sós, ricos em iodo, como: ovo, leite, iogurte, peito de peru, atum, peixe de fígado de bacalhau, feijão da marinha, dentre outros. Há, ainda, alimentos cujo consumo não recomendado para aqueles que apresentam quadro de hipotireoidismo subclínico, como a tapioca (Ovadia 2014, Aakre 2015). Dessa forma, o presente estudo teve como objetivo identificar a influência do zinco, selênio e iodo no controle do hipotireoidismo subclínico.

\section{Metodologia}

Foi realizada uma revisão da literatura. A revisão adota um processo replicável, científico e transparente, sendo recomendada para reunir e analisar os estudos relevantes sobre o tema (Calegari, 2018). Para Kitchenham et al. (2010) a revisão da literatura é uma forma de agregar conhecimento e reunir saberes sobre um tema, e requer um extenso esforço de classificação e encontro de trabalhos relevantes, o que demanda um tempo considerável. É composta por três fases: a) planejamento da revisão, onde identifica-se a necessidade da mesma; b) realizar a revisão, onde identifica-se a a investigação, a seleção dos estudos com avaliação de estudos relevantes, extração dos dados para análise e síntese dos resultados; e, c) relato da revisão (Kitchenham, 2004).

Cinco bases de dados científicas foram utilizadas na pesquisa, ScienceDirect Journals (Elsevier), Directory of Open Access Journals (DOAJ), PMC (PubMed Central), MEDLINE/PubMed (NLM) e Scielo. Os termos indexadores foram utilizados na língua inglesa "hypothyroidism" e "food". Como critério de inclusão foi utilizado artigos que tinham um dos termos indexadores no título e/ou resumo e/ou palavras-chave, publicados nos últimos 5 anos. Como critério de exclusão artigos duplicados e fora do contexto do nosso objetivo. Os resumos dos artigos foram lidos na integra como base para seleção de inclusão ou exclusão. A coleta de dados foi realizada a partir da análise na íntegra dos artigos considerados na revisão. O procedimento foi realizado em setembro de 2020.

\section{Resultados e Discussão}

O processo de busca identificou 70 artigos e após o procedimento de filtragem e seleção resultou em 19 artigos selecionados para o presente estudo. Das cinco bases de dados pesquisadas somente duas apresentaram resultados: Science Direct Journals (Elsevier) e Directory of Open Access Journals (DOAJ), as demais não forneceram nenhum resultado na pesquisa (Tabela 1). 
Tabela 1: Número de artigos encontrados, artigos selecionados e bases de dados pesquisadas.

\begin{tabular}{c|c|c}
\hline BASES DE DADOS & ARTIGOS ENCONTRADOS & $\begin{array}{c}\text { ARTIGOS SELECIONADOS } \\
\text { PARA ESTUDO }\end{array}$ \\
\hline $\begin{array}{c}\text { Science Direct Journals } \\
\text { (Elsevier) }\end{array}$ & 23 & 6 \\
\hline $\begin{array}{c}\text { Directory of Open Access } \\
\text { Journals (DOAJ) }\end{array}$ & 47 & 0 \\
\hline PMC (PubMed Central) & 0 & 0 \\
\hline MEDLINE/PubMed (NLM) & 0 & 0 \\
\hline SCIELO & 0 & \multicolumn{2}{|c}{} \\
\hline
\end{tabular}

Fonte: Autores (2020).

Os artigos selecionados estão representados na Tabela 2 por ordem cronológica do ano de publicação, autor, título do artigo e periódico.

Tabela 2: Artigos selecionados, ano, autor e periódico.

\begin{tabular}{|c|c|c|c|}
\hline ANO & AUTOR & ARTIGO & PERIÓDICO \\
\hline 2019 & BEZERRA,Bianca, et al & $\begin{array}{l}\text { MICRONUTRIENTES ESSENCIAIS PARA } \\
\text { PACIENTES COM HIPERTIREOIDISMO }\end{array}$ & $\begin{array}{l}\text { Journal of Evidence } \\
\text { Based Medicine and } \\
\text { Healthcare }\end{array}$ \\
\hline 2019 & $\begin{array}{l}\text { PHEDORENKO E.V. et } \\
\text { al }\end{array}$ & $\begin{array}{l}\text { RISK COMMUNICATION AS A COMPONENT } \\
\text { THAT PROVIDES STABILITY OF STRATEGY } \\
\text { AIMED AT ELIMINATING DISEASES CAUSED } \\
\text { BY IODINE DEFICIENCY IN BELARUS. }\end{array}$ & $\begin{array}{l}\text { Journal Health Risk } \\
\text { Analysis }\end{array}$ \\
\hline 2019 & EALES J. & $\begin{array}{l}\text { THE RELATIONSHIP BETWEEN } \\
\text { INGESTED THYROID HORMONES, } \\
\text { THYROID HOMEOSTASIS AND IODINE } \\
\text { METABOLISM IN HUMANS AND } \\
\text { TELEOST FISH. }\end{array}$ & $\begin{array}{l}\text { General and comparative } \\
\text { endocrinology }\end{array}$ \\
\hline 2019 & DONNAY S. et al & $\begin{array}{l}\text { DIAGNÓSTICO, TRATAMIENTO Y } \\
\text { MANEJO DEL HIPOTIROIDISMO } \\
\text { GESTACIONAL. ESTUDIO TIROGEST. }\end{array}$ & $\begin{array}{l}\text { Endocrinología, Diabetes } \\
\text { y Nutrición }\end{array}$ \\
\hline 2018 & LYONS G. & $\begin{array}{l}\text { BIOFORTIFICATION OF CEREALS WITH } \\
\text { FOLIAR SELENIUM AND IODINE COULD } \\
\text { REDUCE HYPOTHYROIDISM. }\end{array}$ & Frontiers in Plant Science \\
\hline 2018 & GUSTAVO R. G. et al & $\begin{array}{l}\text { DIETARY SELENIUM INTAKE AND } \\
\text { SUBCLINICAL HYPOTHYROIDISM: A } \\
\text { CROSS-SECTIONAL ANALYSIS OF THE } \\
\text { ELSA-BRASIL STUDY. }\end{array}$ & Nutrients \\
\hline 2018 & PUSZKARZ I. et al & $\begin{array}{l}\text { ROLE OF FOOD AND NUTRITION IN } \\
\text { PATHOGENESIS AND PREVENTION OF } \\
\text { HASHIMOTO'S THYROIDITIS. } \\
\end{array}$ & $\begin{array}{l}\text { Journal of Education, } \\
\text { Health and Sport }\end{array}$ \\
\hline 2018 & YADAV K. et al & 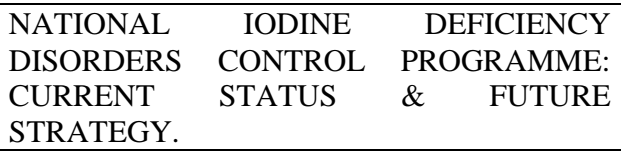 & $\begin{array}{l}\text { Indian Journal of Medical } \\
\text { Research }\end{array}$ \\
\hline 2017 & MAHADEVAN S. et al & $\begin{array}{l}\text { DOES TIME OF } \\
\text { INTAKE ALTER } \\
\text { THYROID } \\
\text { TEST? }\end{array}$ & $\begin{array}{lr}\text { Indian Journal of } \\
\text { Endocrinology } \\
\text { Metabolism }\end{array}$ \\
\hline 2017 & SAMSUDIN M. et al & $\begin{array}{llr}\text { DAMPAK } & \text { INTERVENSI } & \text { GARAM } \\
\text { BERIODIUM } & \text { BERBAGAI } & \text { DOSIS } \\
\text { TERHADAP } & \text { STATUS IODIUM } & \text { DAN } \\
\text { FUNGSI TIROID NORMAL PADA } & \text { ANAK } \\
\text { SEKOLAH DASAR. } & \\
\end{array}$ & $\begin{array}{l}\text { Media } \\
\text { Indonesia }\end{array}$ \\
\hline 2017 & BAHROO S. & $\begin{array}{l}\text { THE NEVER ENDING DEBATE: T3...YES } \\
\text { OR NO? }\end{array}$ & $\begin{array}{ll}\text { Physician } & \text { Assistant } \\
\text { ClinicS } & \\
\end{array}$ \\
\hline 2016 & BALAJEE D. et al & $\begin{array}{l}\text { A STUDY ON CASSAVA [TAPIOCA] } \\
\text { CAUSING HYPOTHYROIDISM. }\end{array}$ & $\begin{array}{l}\text { Journal of Evidence } \\
\text { Based Medicine and } \\
\text { Healthcare }\end{array}$ \\
\hline 2016 & HARBUWONO D. et al & $\begin{array}{lcr}\text { IMPROVEMENT } & \text { OF } & \text { METABOLIC } \\
\text { PARAMETERS } & \text { RESULTED } & \text { FROM } \\
\end{array}$ & Acta Medica Indonesiana \\
\hline
\end{tabular}




\begin{tabular}{|c|c|c|c|}
\hline & & $\begin{array}{llr}\text { LEVOTHYROXINE } & \text { THERAPY } & \text { IN } \\
\text { HYPOTHYROID TYPE } & 2 & \text { DIABETES } \\
\text { MELLITUS PATIENT. } & & \\
\end{array}$ & \\
\hline 2016 & MEZZOMO T. et al & $\begin{array}{l}\text { EFFECT OF NUTRIENTS AND DIETARY } \\
\text { SUBSTANCES ON THYROID FUNCTION } \\
\text { AND HYPOTHYROIDISM }\end{array}$ & $\begin{array}{l}\text { International Journal of } \\
\text { Molecular Sciences }\end{array}$ \\
\hline 2015 & ZELZER S. et al & $\begin{array}{l}\text { OXIDIZED LDL IS STRICTLY LIMITED TO } \\
\text { HYPERTHYROIDISM IRRESPECTIVE OF } \\
\text { FAT FEEDING IN FEMALE SPRAGUE } \\
\text { DAWLEY RATS. }\end{array}$ & $\begin{array}{l}\text { International Journal of } \\
\text { Molecular Sciences }\end{array}$ \\
\hline 2015 & AAKRE I. et al & $\begin{array}{lcr}\text { EXCESSIVE } & \text { IODINE INTAKE } & \text { AND } \\
\text { THYROID } & \text { DYSFUNCTION AMONG } \\
\text { LACTATING SAHARAWI WOMEN. } & \\
\end{array}$ & $\begin{array}{lrr}\text { Journal } & \text { Of } & \text { Trace } \\
\text { Elements In } & \text { Medicine } \\
\text { And Biology } & \end{array}$ \\
\hline 2015 & CHEN Q. et al & $\begin{array}{l}\text { DIETARY METHIMAZOLE-INDUCED } \\
\text { HYPOTHYROIDISM REDUCES HEPATIC } \\
\text { LIPID DEPOSITION BY BOWN- } \\
\text { REGULATING LIPOGENESIS AND UP- } \\
\text { REGULATING } \\
\text { IN PELTEOBAGRUS FULVIDRACO. }\end{array}$ & $\begin{array}{l}\text { General And Comparative } \\
\text { Endocrinology }\end{array}$ \\
\hline 2014 & OVADIA Y. et al & \begin{tabular}{lllr} 
ELEVATED & SERUM & \multicolumn{2}{r}{ THYROGLOBULIN } \\
AND LOW & IODINE & INTAKE & ARE \\
ASSOCIATED & \multicolumn{2}{c}{ WITH } & NONTOXIC \\
NODULAR & GOITER & AMONG & ADULTS \\
LIVING & NEAR & THE & EASTERN \\
MEDITERRANEAN COAST.
\end{tabular} & $\begin{array}{lll}\text { Journal of } & \text { Thyroid } \\
\text { Research } & & \\
\end{array}$ \\
\hline 2014 & BARTON N. & THYROID PREPARATIONS. & $\begin{array}{l}\text { Encyclopedia } \\
\text { Toxicology } \\
\text { Edition) }\end{array}$ \\
\hline
\end{tabular}

Fonte: Autores (2020).

O estado nutricional do organismo é fator fundamental para o bom funcionamento de todos os órgãos no ser humano. Uma dieta adequada e balanceada pode se tornar também um fator importante para ajustar o bom funcionamento da glândula tiróide (GT). Vitaminas e nutrientes minerais são importantes devido ao seu impacto na atividade secretora da tireóide e sua presença é necessária para um bom funcionamento da glândula. Esses nutrientes incluem iodo, ferro, selênio e zinco, bem como vitaminas: A, D, E, B2, B3 e B12. Uma dieta equilibrada permite fornecer todos os nutrientes e minerais necessários, mas existem muitos fatores que podem interferir na adequada absorção desses nutrientes e, como resultado uma suscetível deficiência de vitaminas e minerais necessários.

O iodo é um dos minerais mais importantes que desempenham um papel fundamental no funcionamento da GT, sua deficiência impede a correta produção de hormônios da GT, além de ser essencial para o crescimento e desenvolvimento, particularmente do cérebro e do sistema nervoso central. Sua deficiência é um problema de saúde pública mundial, acometendo cerca de 800 milhões de pessoas. (Triggiani V.et al.,2009). A baixa ingestão deste micronutriente resulta em problemas como o bócio, hipotireoidismo e retardo mental em qualquer fase da vida. Os distúrbios por deficiência de iodo (DDI) são fenômenos amplamente distribuídos em várias regiões do mundo. Populações que vivem em áreas deficientes em iodo sempre terão o risco de apresentar os distúrbios causados por esta deficiência, cujo impacto sobre os níveis de desenvolvimento humano, social e econômico são muito graves. O baixo consumo de alimentos ricos em iodo é a principal causa dos DDI. Os principais alimentos ricos em iodo são os de origem marinha (ostras, moluscos, mariscos e peixes de água salgada); leite e ovos também são fontes de iodo, desde que oriundos de animais que tenham pastado em solos ricos em iodo ou que foram alimentados com rações que continham os nutrientes; vegetais oriundos de solos ricos em iodo também são boas fontes. Uma das orientações para o controle da DDI é a utilização de sal iodado na alimentação. Em vários países ao redor do mundo já é obrigatório por lei à implementação de sal iodado na indústria de alimentos (Ministério da Saúde, 2007).

Desde 1962 a Índia possui programas nacionais voltados para a diminuição da deficiência de iodo. A população indiana é propensa a isso devido à deficiência de iodo no solo. A Iodização Universal de Sal (USI) foi reconhecida como uma 
estratégia fundamental para o controle da deficiência de iodo. Em 1994, a Organização Mundial da Saúde (OMS) e o Comitê Conjunto de Políticas de Saúde do Fundo das Nações Unidas para a Infância (UNICEF) reconheceram a USI como uma estratégia segura, econômica e sustentável para garantir a ingestão suficiente de iodo por todos os indivíduos. A Índia fez um progresso impressionante no controle com a adoção e ampliação bem sucedida da USI no país, o programa de controle na Índia é uma das histórias de sucesso da saúde pública na Índia. A atual cobertura de 92\% no nível doméstico de sal iodado na Índia. Outros países como a Bielorrússia o sal iodado também é adicionado na indústria alimentícia como estratégia para diminuição da DDI e apresenta resultados satisfatórios (Yadav 2018, Phedorenko 2019).

Debates sobre a dosagem adequada de sal iodado são bastante frequentes, pois a excesso de iodo no organismo também é prejudicial a GT e ainda não existem estudos suficientes que definam a dosagem eficaz do uso do sal iodado. A sobrecarga iodada de origem alimentar é raríssima. A maioria das pessoas pode tolerar, pelo menos, $1 \mathrm{mg}(1000 \mathrm{mcg})$ de iodo por dia sem efeitos adversos. A maioria das pessoas com um aporte prévio de iodo suficiente, toleram facilmente grandes quantidades de iodo no organismo. Existem algumas situações onde podem existir danos provocados pelo excesso de iodo tais como o hipertiroidismo induzido pelo excesso de iodo nos indivíduos com doença nodular da tiróide e o hipotiroidismo por bloqueio da capacidade da glândula em produzir hormonas. Indivíduos com doenças auto-imunes da tiróide, como a doença de Graves ou tiroidite de Hashimoto, ou com familiares com estas patologias também podem ser mais sensíveis ao iodo. A alta ingestão de iodo numa população está associada a um aumento da incidência destas doenças auto-imunes da tiróide. Foi descrito um aumento da incidência de carcinoma papilar da tiróide em zonas de elevado aporte de iodo, embora esta situação não esteja ainda bem esclarecida.15 O excesso de iodo é indesejável, mas as suas consequências não são tão graves como as do défice de iodo, porque estas afetam o desenvolvimento humano e podem provocar danos cerebrais permanentes. (Lopes 2012).

De acordo com Lopes (2012) o sal iodado raramente vai adicionar mais de $300 \mu \mathrm{g}$ de iodo por dia à dieta. Portanto, a preocupação com o excesso de iodo, não é uma razão para suspender ou evitar o consumo de sal iodado. Realizou um estudo onde teve como objetivo definir a dosagem ideal de sal iodado, em crianças do ensino fundamental no subdistrito de Kepil, distrito de Wonosobo por dez meses. Foram 176 crianças, com idades entre 6 e 12 anos. A intervenção com sal iodado (KIO3) de 15-55 ppm por quatro meses geralmente era capaz de fornecer iodo adequado e uma função tireoidiana normal em crianças do ensino fundamental.

Outro fator importante observado no presente estudo foi relacionado à informação da população sobre a importância da suplementação de Iodo. Phedorenko (2019) realizou uma pesquisa e teve como um de seus objetivos avaliar a conscientização da população sobre a deficiência de iodo. A pesquisa foi realizada na Bielorrússia, onde por lei é implementado sal iodado de alta qualidade na indústria de alimentos a fim de reduzir problemas relacionados à deficiência de iodo. Foi identificado que tanto a população quanto a classe médica tem pouca ou nenhuma conscientização sobre a importância dessa suplementação na saúde (Phedorenko 2019).

O próximo elemento necessário para uma dieta de pacientes com hipotireoidismo é o Ferro. É um ingrediente da peroxidação da tireóide, uma enzima que participa da síntese de hormônios da glândula tireóide. Falta de ferro causa redução da síntese de hormônios tireoidianos através da conversão reduzida de T4 em T3 e contribui para o aumento da secreção de TSH (Puszkarz 2019).

Na tireoide destaca-se como um dos órgãos com maior concentração de selênio, o qual está principalmente presente na estrutura molecular de selenoproteínas envolvidas no metabolismo dos hormônios tireoidianos e na proteção antioxidante da glândula (Aaseth Et Al., 1990; Dickson; Tomlinson, 1967; Drutel; Archambeaud; Caron, 2013).

O selênio é um elemento-traço importante para os mecanismos antioxidantes, para o sistema imunológico, e ainda participa ativamente da homeostase da glândula tireoide. Uma vez que o este mineral, como selenocisteína, é um cofator para a 5'D, tem recebido muita atenção no que diz respeito ao metabolismo periférico dos HT. Como citado anteriormente, a enzima 
5’D hepática converte a T4 em T3r ou em T3 ativo. Se há deficiência de selênio, a atividade das deiodinases (ID) será prejudicada. A glândula tireoidiana normal retém altas concentrações de selênio e expressa muitas das selenocisteínas. Estas são encontradas no centro catalítico de enzimas que protegem a tireoide de danos de radicais livres. A deficiência de selênio tem sido um achado constante em doenças da glândula tireoide. Nas regiões com deficiência grave de iodo combinada com a de selênio, a normalização do fornecimento de iodo é obrigatória antes do início da suplementação de selênio, no sentido de impedir o hipotireoidismo. Desta forma, percebe-se que o selênio desempenha papel importante na regulação da função da tireoide, bem como na homeostase de HTs, através da ação de selenoproteínas, em que se incorpora como selenocisteína e, por último, protege a glândula dos danos causados pela exposição excessiva de iodeto. Carnes e frutos do mar são ótimas fontes de selênio, bem como a castanha do Brasil (Mezzomo 2016, Lyons 2018, Andrade 2018).

Contudo, a concentração de selênio nas castanhas varia de acordo com a capacidade de absorção da árvore e com a

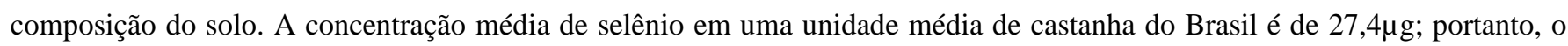
consumo de duas unidades diárias é suficiente para abranger a RDA.36 O teor de selênio nas carnes e pescados varia entre 2,8 a $80,9 \mu \mathrm{g} / 100 \mathrm{~g}$ de alimento, sendo os pescados (atum e sardinha, principalmente) os alimentos com maior quantidade deste mineral.37 O feijão preto e a farinha de trigo integral também apresentam quantidades importantes de selênio, sendo 11,9 $\mu \mathrm{g} / 100 \mathrm{~g}$ e 13,6 $\mu \mathrm{g} / 100 \mathrm{~g}$, respectivamente (Mezzomo 2016, Lyons 2018, Andrade 2018).

O zinco possui propriedades antioxidantes e anti-inflamatórias é um nutriente mineral subsequente que participa do bom funcionamento do sistema imunológico. Quanto menor a concentração de zinco no soro sanguíneo, maior a presença de anticorpos anti-tireoidianos, que podem confirmar o papel do zinco no funcionamento da defesa imunológica do organismo. Este elemento é um componente das proteínas receptoras T3, portanto, sua deficiência afeta o hormônio prejudicado ligação, o que resulta em concentração reduzida de T3 e T4 (Puszkarz 2019). Há relatos, na literatura, de que a deficiência de zinco é uma causa de hipotireoidismo subclínico. Em estudos com animais, a deficiência de zinco resultou em diminuição de aproximadamente 30\% nos níveis de T3 e T4 livres. Em seres humanos, a suplementação de zinco restabeleceu a função tireoidiana normal em pacientes com hipotireoidismo. Esse nutriente pode ser encontrado em carnes e pescados. Mais estudos são necessários para esclarecer o papel do zinco na função tireoidiana (Mezzomo 2016).

\section{Conclusão}

Os minerais são importantes para a manutenção do organismo e para a produção de hormônios tireoidianos, com isso as quantidades destes componentes no corpo devem ter sempre um equilíbrio.

O iodo tem papel fundamental na produção dos hormônios da Tireoide. Quantidades excessivas ou deficitárias de iodo e selênio contribuem para alterações tireoidianas, entre as quais o hipotireoidismo. O selênio e o zinco agem como cofatores nas reações de deiodinação do T4 em hormônio ativo. São necessários mais estudos para avaliar a real ingestão de iodo e selênio pela população brasileira, com a finalidade de evitar sua deficiência ou ainda, seu excesso.

A influência dos nutrientes na função tireoidiana ainda é um tanto quanto nebulosa para a ciência, fato este que revela a necessidade de estímulo a novas pesquisas no sentido de ampliar o conhecimento nessa área, para que medidas preventivas ou de apoio ao tratamento possam ser tomadas e auxiliem efetivamente na adequação do funcionamento da tireoide.

O sistema imune é o principal responsável por impedir o funcionamento normal da tireoide, quanto à produção de hormônios, fundamentais ao metabolismo humano normal, principalmente quando o iodo, selênio e zinco estão em concentrações inadequadas;

Sugere-se que outros artigos ou trabalhos sejam realizadas a fim de que seja verificada a possível interferência de outros nutrientes ou substâncias alimentares na função tireoidiana. 
Research, Society and Development, v. 10, n. 16, e268101623719, 2021

(CC BY 4.0) | ISSN 2525-3409 | DOI: http://dx.doi.org/10.33448/rsd-v10i16.23719

\section{Referências}

Aaseth, J. et al. (1990). Selenium concentrations in the human thyroid gland. Biol Trace Elem Res. 24(02),147-52.

Aakre I. et al. Excessive iodine intake and thyroid dysfunction among lactating Saharawi women. J Trace Elem Med Biol. 31:279-84.

Andrade, G., Gorgulho, B., Lotufo, P., Bensenor, I., \& Marchioni, D. (2018). Dietary Selenium Intake and Subclinical Hypothyroidism: A Cross-Sectional Analysis of the ELSA-Brasil Study. Nutrients. 10. 693. 10.3390/nu10060693.

Brenta, Gabriela et al .(2013). Diretrizes clínicas práticas para o manejo do hipotiroidismo. Arq bras endocrinol metab, 57(4), 265-291.

Calegari L. P., \& Fettermann D. C. (2018) A systematic review to identify possible applications and barriers to the adoption of mass customization in food production. Braz. J. Food Technol., 21, 2017096, 2018.

Chen Q. L. et al. Dietary Methimazole-Induced Hypothyroidism Reduces Hepatic Lipid Deposition By Down-Regulating Lipogenesis And Up-Regulating Lipolysis In Pelteobagrus Fulvidraco. Gen Comp Endocrinol. 28-36.

Deficiência em Iodo - Dicas em Saúde. BVS. Ministério da Saúde. Coordenação-Geral da Política de Alimentação e Nutrição - CGPAN. 2007.

Dickson, R. C., \& Tomlinson, R. H. (1967) . Selenium in blood and human tissues. Clin Chim Acta. 16(02), 311-21.

Drutel, A., Archambeaud, F., \& Caron, P. (2013). Selenium and the thyroid gland: more good news for clinicians. Clin Endocrinol (Oxf). 78(02), 155-64.

Kitchenham, B, et al. (2010). Systematic literature reviews in software engineering: a tertiary study. Information \& Software Technology, 52(8), 792-805.

Kitchenham, B. (2004). Procedures For Performing Systematic Reviews. KEELE, 33, 1-26.

Lopes, M., Castro, J., Marcelino, M. Oliveira, M., Carrilho, F., \& Limbert, E. (2012). Iodine and Thyroid: What a Clinic Should Know. Acta médica portuguesa. $25.174-8$.

Lyons, G. (2018). Biofortification of Cereals With Foliar Selenium and Iodine Could Reduce Hypothyroidism. Frontiers in Plant Science. 9. 730. 10.3389/fpls.2018.00730.

Mahadevan S., Sadacharan D., Kannan S., Suryanarayanan A. Does time of sampling or food intake alter thyroid function test? Indian J Endocr Metab 21:369-72

Mezzomo, T., \& Nadal, J. (2016). Efeito Dos Nutrientes e Substâncias Alimentares Na Função Tireoidiana E No Hipotireoidismo. Demetra: Alimentação, Nutrição \& Saúde. 11. 10.12957/demetra.2016.18304.

Ovadia, Y. S et al. "Elevated Serum Thyroglobulin and Low Iodine Intake Are Associated with Nontoxic Nodular Goiter among Adults Living near the Eastern Mediterranean Coast." Journal of thyroid research vol. 2014 (2014): 913672. doi:10.1155/2014/913672.

Pearce, E. N. Iodine deficiency in children. Endocr. Dev. 2014, 26:130-8.

Phedorenko, E. V. Kolomiets, N. D. Mokhort, T. V. Volchenko, A. N. Mokhort, E. G. Petrenko, S. V. \& Sychik, S. I. (2019). Risk communication as a component that provides stability of strategy aimed at eliminating diseases caused by iodine deficiency in Belarus. Health Risk Analysis. 58-67. 10.21668/health.risk/2019.1.06.eng.

Puszkarz, I. et al. (2018). Role of food and nutrition in pathogenesis and prevention of Hashimoto's thyroiditis. Journal of Education, Health and Sport, [S.1.].8(7),394-401. http://www.ojs.ukw.edu.pl/index.php/johs/article/view/5702.

Triggiani V, Tafaro E, Giagulli V. A, Sabbà C, Resta F, Licchelli B, et al. Role of iodine, selenium and other micronutrients in thyroid function and disorders. Endocr. Metab. Immune Disord. Drug Targets 2009, 9(3):277-94.

Yadav K., \& Pandav C. S. (2018). National Iodine Deficiency Disorders Control Programme: Current status \& future strategy. Indian J Med Res. 2018,148(5):503-510. doi:10.4103/ijmr.IJMR_1717_18. 